\title{
La Odontología en Mexico Durante la Pandemia de COVID-19
}

\author{
Dentistry in Mexico During the COVID-19 Pandemic
}

\author{
Acosta Rangel M. ${ }^{1}$; Flores Meza B.. ${ }^{2}$ \& Delgado Galindez B. ${ }^{3}$
}

\begin{abstract}
ACOSTA, R. M.; FLORES, M. B \& DELGADO, G. B. La odontología en Mexico durante la pandemia de COVID-19. Int. J. Odontostomat., 15(3):666-669, 2021.

RESUMEN: Este artículo informa las características de la pandemia que en la actualidad estamos enfrentando y enfatiza la importancia de la rigurosidad con las medidas de bioseguridad y el cumplimiento cabal de las normas oficiales mexicanas al respecto; en la práctica de la atención dental. Es un trabajo de revisiones sistemáticas sobre los mecanismos de protección usados en la práctica odontológica a nivel internacional y nacional, así como las medidas que se han implementado a nivel nacional e internacional y la importancia de la atención odontológica general en tiempos de pandemia derivado de COVID -19. Así mismo la importancia en esta situación tan compleja de tomar como pauta la necesidad de tener números reales del personal que labora en el país, y las condiciones en las cuales se lleva a cabo la práctica, esto hace un parteaguas dentro de nuestros tiempos y en nuestra área de genera informacion fidedigna y puntual sobre las condiciones que prevalecen en este personal de salud tan relevante e importante para la salud en esta área.
\end{abstract}

PALABRAS CLAVE: pandemia, COVID-19, contagio, atención dental.

\section{INTRODUCCIÓN}

Actualmente estamos viviendo momentos críticos y de replanteamiento sobre nuestra práctica clínica diaria, al coexistir con un virus sumamente contagioso y para algunos letal, por lo que debemos, como personal del área de la salud involucrados en la atención, tanto en odontología como en diversos procedimientos quirúrgicos, el estar bien informados y capacitados sobre el tema.

La práctica odontológica está sufriendo una transformación y cambios en la conducta para la atención del paciente en instituciones públicas y privadas. Las medidas de bioseguridad deben llevarse a cabo con rigurosidad, identificando los factores de riesgo en la práctica odontológica; debemos preocuparnos, pero sobre todo ocuparnos de la protección de las áreas de trabajo, del paciente y desde luego del personal de la salud en la atención integral del paciente odontológico; minimizando el riesgo de infección ya que el área odontológica es de alto riesgo por la producción de aerosoles que se producen en la práctica cotidiana.

Este artículo informa las características de la pandemia que en la actualidad estamos enfrentando y enfatiza la importancia de la rigurosidad con las medidas de bioseguridad y el cumplimiento cabal de las normas oficiales mexicanas al respecto; en la práctica de la atención dental.

Salud bucal. En la salud bucal se trabaja muy cerca al rostro de los pacientes durante períodos prolongados de tiempo en cada cita, sus procedimientos implican comunicación cara a cara y exposición frecuente a la saliva, sangre, secreciones nasales, sudor, detritus y manipulación de instrumentos cortantes. En consecuencia, el personal médico y paramédico tiene un alto riesgo de infectarse con el virus del SARS-CoV-2 o transmitir la infección a los pacientes (WHO, 2020).

\footnotetext{
${ }^{1}$ Hospital General, Centro Médico Nacional La Raza, Médico Adscrito al Servicio de Cirugía Maxilofacial Pediátrica, México.

${ }^{2}$ Cirujano de Cabeza y Cuello, Otorrinolaringólogo Pediátrico, Mtra. En Ciencias de la Salud México.

${ }^{3}$ Cirujano Oral y Maxilofacial, Mtra. En administración de servicios de salud México.
} 
La OMS aconseja que la atención de salud bucodental no esencial de rutina, que generalmente incluye controles de salud bucal, limpiezas dentales y atención preventiva, hay que retrasarla hasta que haya suficiente reducción en las tasas de transmisión de COVID-19 de la comunidad, transmisión a casos de clúster o de acuerdo con recomendaciones a nivel nacional, estatal y/o local (ADA, 2021).

México no cuenta con comités, asociaciones o algún órgano que regule, rija y establezca parámetros que hagan cumplir estas recomendaciones para una práctica segura, hasta ahora todo lo que se ha implementado ha sido a partir de recomendaciones de otros países, sin existir una política o regulación nacional en el establecimiento de nuevas pautas, en la práctica durante la contingencia de salud, incluso el contar con los equipos de protección ha sido un camino complejo para el personal del área odontológica, que tampoco se ha capacitado para la protección de sí mismo y no existen lineamientos normados para un practica segura en el consultorio y en el paciente.

Sin embargo, la OMS como parte de las medidas para disminuir los riesgos en los consultorios o clínicas, señala puntos a considerar como son las áreas ventiladas, el tipo de ventilación que se deberá tener para mejorar las condiciones internas de los establecimientos dentales.

\section{Ventilación en entornos de salud oral}

La ventilación adecuada en los establecimientos de salud bucal reduce el riesgo de transmisión en entornos cerrados. De acuerdo con el tipo de ventilación disponible (mecánica o natural), aumente la ventilación y el flujo de aire: puerta abierta, ventilación de extracción adecuada, presión negativa o capacidad de intercambio de aire equivalente ventilado mecánicamente en la habitación cuando sea posible (promedio de 6 - 12 intercambios de aire por hora), mejor conocidos como extractores de aire.

Evitar el uso de aire acondicionado split u otros tipos de dispositivos de recirculación y considerar la instalación de sistemas de filtración. Se pueden deliberar los siguientes enfoques: instalación de extractores de aire; instalación de remolinos (por ejemplo: remolinos, turbinas eólicas) o instalación de filtros de aire de partículas de alta eficiencia (HEPA).

Cualquier modificación a la ventilación de la instalación de salud bucal debe realizarse con cuidado, teniendo en cuenta el costo, el diseño, el mantenimiento y el impacto potencial en el flujo de aire en otras partes de la instalación (WHO, 2021a).

Transmisión de COVID-19 en entornos de atención de salud bucal. Los procedimientos de generación de aerosoles (AGP) se realizan ampliamente en todo el mundo en entornos de atención de la salud bucal. Los AGP se definen como cualquier procedimiento médico, dental y de atención al paciente que resulte en la producción de partículas en el aire de $<5$ micrómetros ( $\mathrm{mm}$ ) en tamaño (aerosoles), que pueden permanecer suspendidos en el ambiente, viajara distancia y logran causar infección si se inhalan. Los equipos generadores de pulverizaciones, como la pieza de mano de alta velocidad, la pieza triple y el aspirador, provocan la formación de aerosoles en el área de tratamiento, lo que lleva a una rápida contaminación de las superficies (CDCP, 2020; Ge, 2020).

La transmisión del SARS-CoV-2, el virus que causa COVID-19, puede ocurrir a través del contacto directo, indirecto o cercano con personas infectadas a través de secreciones como saliva y secreciones respiratorias o por sus gotitas respiratorias, que tienen < 5 - $10 \mathrm{~mm}$ de diámetro. Gotas de $<5 \mathrm{~mm}$ de diámetro se conocen como núcleos de gotitas o aerosoles (CDCP).

Para más información reciente sobre la transmisión del virus, enlace a"Transmisión del SARS-CoV2: implicaciones para la infección precauciones de prevención (WHO, 2020b).

Se realizó una revisión sistemática y un metaanálisis (Chu et al., 2020) con el objetivo de conocer la distancia persona-persona óptima y el efecto del uso de mascarillas faciales N95 y protección ocular en la disminución de la transmisión. Se graduó la calidad de la evidencia según el sistema GRADE. EI riesgo de transmisión del virus disminuye con el distanciamiento físico de 1 metro (OR: 0,18 IC $95 \%$ 0,09 - 0,38, RA - 10,2 \%, IC 95\% - 11,5\% a - 7,5\%, (calidad de evidencia moderada). La disminución del riesgo aumenta conforme aumenta la distancia con un cambio en el RR de 2.02 por metro extra ( $p=0,041$, calidad de evidencia moderada).

El uso de mascarillas faciales N95 resulta en una mayor disminución del riesgo de infección (OR: 0,15 IC $95 \%$ 0,07 - 0,34, RA - 14.3\%, IC $95 \%$ - 15,9 $\%$ a $-10,7 \%$, calidad de evidencia baja). 
El uso de protección ocular también se asocia a una disminución en el riesgo de contagio (OR: 0,22 IC $95 \% 0,12-0,39$, RA - 10,6\%, IC $95 \%-12$ - $5 \%$ a $7,7 \%$, calidad de evidencia baja).

En conclusión, podemos decir que: se obtuvo evidencia suficiente para apoyar el distanciamiento físico mínimo de un metro, que podríamos aplicar, por ejemplo, en las salas de espera, tanto en la práctica institucional como en la privada. El uso de mascarillas faciales N95 para los médicos y personal paramédico y de protectores oculares, por lo tanto se debe recomendar siempre que sea de manera conveniente y apropiada en cada contexto particular, además de seguir en la espera de nuevas publicaciones con datos científicos que lo respalden, y aunque es necesario más estudios aleatorizados para una mejor estimación del efecto de estas medidas, esta evidencia es la mejor con la que se cuenta al momento y debe ser la guía para las recomendaciones actuales y aún más, en el caso del área odontológica, por el potencial riesgo que se somete al personal médico y paramédico a los aerosoles y sin dejar de lado también, el destacado papel de los guantes de látex en la prevención y protección, así como por supuesto, el lavado de manos, antes, durante y al final de cada procedimiento.

El estomatólogo está invariablemente en contacto con la saliva, que se considera la responsable de la mayoría de las infecciones. COVID-19 que se transmite principalmente de tres formas en la salud bucal en los entornos de atención:

1) Transmisión directa a través de la inhalación de gotitas generadas al toser o estornudar.

2) Transmisión indirecta a través de la exposición de la membrana mucosa como el ojo, mucosa nasal u oral a gotitas infecciosas.

3) Transmisión indirecta a través de superficies contaminadas (Peng et al., 2020).

Debido al alto riesgo de exposición al coronavirus por parte de los dentistas, es urgente establecer medidas laborales seguras, considerando la viabilidad de los sistemas de salud y la preservación de la salud de pacientes y profesionales (Carrer et al., 2020).

\section{DISCUSIÓN}

Es prioritario contar con guías de manejo o pautas que permitan en nuestro país el apego a las mejo- res prácticas y seguridad para la consulta en el ámbito odontológico es lo ideal, ya que la salud dental representa un reto para nuestro nación, la OMS público un artículo recientemente en agosto 2020 en el cual señala la necesidad de dichas normas, esta guía provisional está destinada a las autoridades de salud pública, jefes de odontología en ministerios de salud y personal de salud bucal que trabaja en Sectores de salud pública y privada (WHO, 2021b). Por lo que se deberán de aplicar estrategias de información virtual, considerando esta situación actual de sana distancia, tanto para pacientes y personal involucrado en la atención dental, ya que la prevención eficaz del cuidado estomatológico, los problemas y el cuidado personal, siguen siendo una alta prioridad. Los pacientes deben recibir asesoramiento a través de consultas remotas o por redes sociales como canales de trasmisión sobre el mantenimiento de una buena higiene de la cavidad oral.

\section{CONCLUSIONES}

Es importante el mostrar un apego adecuado y actualizado a esta nueva realidad en el manejo del paciente en la consulta y los diferentes procedimientos quirúrgicos, tanto en la práctica pública como en privada, y en las escuelas o facultades odontológicas, donde se lleva a cabo la enseñanza de esta profesión, apoyándose en publicaciones recientes y con un buen sustento científico sobre el uso de una ventilación apropiada y del Equipo de Protección Personal (EPP) idóneo como la mascarilla, lentes, guantes y por supuesto incluyendo el lavado apropiado de manos. Incluso no existe en este momento un censo que determine la cantidad de odontologos de practica pública y privada, así como en este rubro si este personal de la práctica privada esta titulada y en su consulta utiliza las medidas mínimas de prevención y / o si existen las condiciones en sus consultorios para una práctica segura para ellos y los pacientes que se tratan.

Así mismo la importancia en esta situación tan compleja de tomar como pauta la necesidad de tener números reales del personal que labora en el país, y las condiciones en las cuales se lleva a cabo la práctica, esto hace un parte aguas dentro de nuestros tiempos y en nuestra área degenera información fidedigna y puntual sobre las condiciones que prevalecen en este personal de salud tan relevante e importante para la salud en esta área. 
ACOSTA, R. M.; FLORES, M. B2 \& DELGADO, G. B. Dentistry in Mexico during the covid-19 pandemic. Int. J. Odontostomat., 15(3):666-669, 2021.

ABSTRACT: This article reports on the characteristics of the pandemic that we are currently facing and emphasizes the importance of rigor with biosafety measures and full compliance with official Mexican standards in this regard; in the practice of dental care. It is a work of systematic reviews on the protection mechanisms used in dental practice at the international and national level, as well as the measures that have been implemented at the national and international level and the importance of general dental care in times of pandemic derived from COVID -19. Likewise, the importance in this complex situation of taking as a guideline the need to have real numbers of personnel working in the country. In addition, the conditions in which the practice is carried out, consistent with our times and our area, and ensure that accurate and timely information on the conditions that prevail in these health personnel, which is relevant to health in this area.

KEY WORDS: pandemic, COVID-19, contagion, dental care.

\section{REFERENCIAS BIBLIOGRÁFICAS}

American Dental Association (ADA). What constitutes a dental emergency?. American Dental Association, 2021. Disponible en: https://success.ada.org/ /media/CPS/Files/Open\%20Files/ ADA_COVID19_Dental_Emergency_DDS.pdf

Carrer, F.; Galante, M. L.; Gabriel, M.; Pischel, N.; Giraldes, A. I.; Neumann, A.; da Silva, D. P. \& Pucca, G. A. A COVID-19 na América Latina e suas repercussões para a odontologia. Rev. Panam. Salud Publica, 44:e66, 2020.

Centers for Disease Control and Prevention (CDCP). Interim infection prevention and control guidance for dental settings during the COVID-19 response. Centers for Disease Control and Prevention, 2020. Disponible en: https:// www.fdiworlddental.org/interim-infection-prevention-and-control-guidance-dental-settings-during-covid-19-response

Chu, D. K.; Akl, E. A.; Duda, S.; Solo, K.; Yaacoub, S.; Schünemann, H. J. \& COVID-19 Systematic Urgent Review Group Effort (SURGE) study authors. Physical distancing, face masks, and eye protection to prevent person-to-person transmission of SARS-CoV-2 and COVID-19: a systematic review and metaanalysis. Lancet (London, England), 395(10242):1973-87, 2020.

Ge, Z. Y:, Yang, L. M.; Xia, J. J.; Fu, X. H. \& Zhang, Y. Z. Possible aerosol transmission of COVID-19 and special precautions in dentistry. J. Zhejiang. Univ. Sci. B., 21(5):361-8, 2020.

Peng, X.; Xu, X.; Li, Y.; Cheng, L.; Zhou, X. \& Ren, B. Transmission routes of 2019-nCoV and controls in dental practice. Int. J. Oral Sci., 12(1):9, 2020.

World Health Organization (WHO). Considerations for the provision of essential oral health services in the context of COVID-19. World Health Organization. 2020a. Disponible en: file:///Users/ belgicavasquez/Downloads/WHO-2019-nCoV-Oral_health2020.1-eng.pdf
World Health Organization (WHO). Modes of transmission of virus causing COVID-19: implications for IPC precaution recommendations. World Health Organization, 2020b. Disponible en: https://reliefweb.int/sites/reliefweb.int/files/resources/ WHO-2019-nCoV-Sci_Brief-Transmission_modes-2020.1eng.pdf

World Health Organization (WHO). Infection prevention and control during health care when coronavirus disease (COVID-19) is suspected or confirmed: Interim guidance. World Health Organization, 2021a. Disponible en: https://www.who.int/ publications/i/item/WHO-2019-nCoV-IPC-2021.1

World Health Organization (WHO). Oral health. World Health Organization, 2021b. Disponible en: https://www.who.int/healthtopics/oral-health

Dirección para Correspondecia:

Mónica C. Acosta Rangel

Cirujano Maxilofacial Pediátrica

Hospital General Centro Médico Nacional La Raza

Médico Adscrito al Servicio de Cirugía Maxilofacial Pediátrica Cirujano Maxilofacial Pediátrica

MÉXICO

E-mail: :acora_m@hotmail.com 